\title{
microRNA-1273g-3p is a useful non-invasive test for the prediction of liver fibrosis in patients with chronic hepatitis $\mathbf{C}$
}

\author{
YUEMIN NAN, XUEMIN NIU, RONGQI WANG, SUXIAN ZHAO, NA FU, \\ JINGHUA DU, YANG WANG, BAOYU WANG and YUGUO ZHANG \\ Department of Traditional and Western Medical Hepatology, \\ Third Hospital of Hebei Medical University, Shijiazhuang, Hebei 050051, P.R. China
}

Received October 14, 2016; Accepted July 7, 2017

DOI: $10.3892 /$ etm.2018.7114

\begin{abstract}
Previous studies using microRNA (miRNA or miR) microarrays have demonstrated that miR-1273g-3p is upregulated in patients with hepatitis $\mathrm{C}$ virus ( $\mathrm{HCV}$ )-associated fibrosis. As miRNAs have been suggested to be promising non-invasive biomarkers, the aim of the present study was to assess whether miR-1273g-3p may be useful as a potential indicator of fibrosis progression in patients with HCV. Liver biopsies were performed on 112 patients with chronic hepatitis $\mathrm{C}(\mathrm{CHC})$ and liver stiffness measurements (LSM) were performed using FibroTouch. Liver fibrosis was determined based on Meta-analysis of Histological Data in Viral Hepatitis classification, and the aspartate aminotransferase (AST)-to-platelet count (PLT) ratio index (APRI) and Fibrosis-4 score (FIB-4) were calculated. The diagnostic performance of miR-1273g-3p, LSM, APRI and FIB-4 in predicting fibrosis stage were evaluated and compared by receiver operating characteristic (ROC) analysis. It was demonstrated that miR-1273g-3p levels were significantly positively correlated with the liver fibrosis stage $(r=0.657, \mathrm{P}<0.001)$. The results of LSM, APRI and FIB-4, the three non-invasive diagnostic methods, had good consistency with liver biopsy results, and their correlation coefficients with fibrosis staging were 0.815 , 0.417 and 0.522 , respectively. The areas under the ROC curves of miR-1273g-3p for $\mathrm{F} \geq 2$ and $F=4$ stage samples were 0.841 and 0.933 , respectively, which were lower than LSM $(0.890$ and 0.937), and higher than FIB-4 (0.791 and 0.766) and APRI (0.719 and 0.760). Spearman analysis demonstrated that serum miR-1273g-3p levels were significantly positively correlated with age, body mass index, alanine aminotransferase, AST
\end{abstract}

Correspondence to: Professor Yuemin Nan, Department of Traditional and Western Medical Hepatology, Third Hospital of Hebei Medical University, 139 Ziqiang Road, Shijiazhuang, Hebei 050051, P.R. China

E-mail: nanyuemin@163.com

Key words: hepatic fibrosis, chronic hepatitis $\mathrm{C}$ virus infection, microRNA-1273g-3p, liver stiffness measurements, aspartate aminotransferase to platelet count ratio index, fibrosis-4 score and total bilirubin (all $\mathrm{P}<0.05$ ), and negatively correlated with PLT $(\mathrm{P}<0.05)$. However, no significant correlation was observed between miR-1273g-3p levels, baseline HCV RNA loads and genotype. Therefore, the results demonstrated that miR-1273g-3p levels, as a novel non-invasive test, may be a useful and easy method for predicting the stage of liver fibrosis in patients with $\mathrm{CHC}$, and has a better diagnostic performance than FIB-4 and APRI. Further prospective studies are required to validate the efficacy of miR-1273g-3p as a predictor of liver fibrosis.

\section{Introduction}

Hepatitis $\mathrm{C}$ virus (HCV) is considered to be one of the major causes of liver fibrosis and cirrhosis. Estimating the stage of disease is essential for determining disease prognosis and the appropriate antiviral therapy (1). Although therapy is not essential to treat insignificant fibrosis [Meta-analysis of Histological Data in Viral Hepatitis (METAVIR stage F1)] in patients with chronic hepatitis $\mathrm{C}$ (CHC), significant fibrosis (METAVIR stage $\mathrm{F} \geq 2$ ) must be treated to avoid progression to cirrhosis or hepatocellular carcinoma (HCC) (2-4). Therefore, diagnosis of the fibrosis stage is crucial in routine clinical practice. Liver biopsy is the gold standard method for the staging of fibrosis; however, this invasive technique has a mortality rate of $0.01-0.1 \%$ and harbors the risk of severe complications (5). Therefore, accurate, non-invasive and readily available methods for identifying fibrosis in patients with HCV are urgently required.

In the past decade, microRNAs (miRNAs or miRs) have been proposed as useful biomarkers for predicting the presence and severity of various pathologies $(6,7)$. Numerous studies have identified specific miRNAs that serve important roles in a variety of cellular processes, including metabolism, immune function, cell proliferation and apoptosis (8-12). The circulating miRNA profile is altered during the initiation and progression of various liver diseases (13), and there are correlations between circulating miRNA levels and various clinicopathological endpoints $(14,15)$. This, in addition to the remarkable stability of miRNAs in plasma, serum and other body fluids, emphasizes their significance as a novel class of blood-based biomarkers and offers novel strategies for the development of non-invasive prognostic tests (16-18). 
Various non-invasive diagnostic and prognostic methods, ranging from serum biomarker assays to advanced imaging techniques, are being developed $(19,20)$. Analysis of liver stiffness measurements (LSM), the aspartate aminotransferase (AST)-to-platelet (PLT) ratio index (APRI) and fibrosis 4 score (FIB-4) are now routinely performed to assess fibrosis in patients with liver disease $(12,21)$. The majority of these non-invasive tests are primarily used to distinguish the presence of cirrhosis from minimal or absent fibrosis; however, they have a low diagnostic performance, particularly for the diagnosis of significant fibrosis (19). These non-invasive tests are also affected by the weight of the patient, the presence of ascites, and the transaminase and bilirubin levels (22-24).

The aim of the present study was to evaluate the performance of miR-1273g-3p levels as a diagnostic tool for fibrosis (METAVIR stage $\mathrm{F} \geq 2$ and $\mathrm{F}=4$ ), using liver histology as the gold standard for diagnosis. Additionally, the diagnostic performance of miR-1273g-3p was compared with LSM, APRI and FIB-4, and the value of using miR-1273g-3p to predict the fibrosis stage in patients with CHC was also determined.

\section{Materials and methods}

Patients. A total of 112 patients (56 males and 56 females) with $\mathrm{CHC}$ infection underwent liver biopsies and provided blood samples at the Third Hospital of Hebei Medical University (Shijiazhuang, China) from January 2014 to May 2016. CHC infection was diagnosed on the basis of positive tests for serum antibodies against $\mathrm{HCV}$ and the presence of HCV RNA in the plasma in the previous 6 months. Eligible patients were $>18$ years of age. Patients with any of the following were excluded from the present study: Presence of decompensated cirrhosis, co-infection with human immunodeficiency virus, hepatitis $\mathrm{A}, \mathrm{B}$ or $\mathrm{D}$ virus infection, other causes of chronic liver disease or co-morbidities precluding interferon therapy $(25,26)$. Written informed consent was obtained from all patients, and the study was approved by the Ethics Committee of the Third Hospital of Hebei Medical University, according to the Declaration of Helsinki and Good Clinical Practice guidelines.

Detection of antibodies, viral load and genotypes of $\mathrm{HCV}$. Serum HCV antibodies were detected using commercial anti-HCV ELISA kits (cat. nos. 2013040508, 2013111208, 2014030608 and 2014081408; Zhuhai Livzon Diagnostics, Inc., Zhuhai, China), which are approved by China's State Food and Drug Administration (SFDA: S10950020). Plasma HCV RNA was determined by reverse transcriptionquantitative polymerase chain reaction (RT-qPCR) using a COBAS TAQman HCV test (Roche Molecular Diagnostics, Pleasanton, CA, USA), which included three major processes: specimen preparation to isolate HCV RNA; reverse transcription of the target RNA to generate cDNA and simultaneous PCR amplification of target cDNA, and detection of cleaved dual-labeled oligonucleotide detection probes specific to the target. The lowest limit of quantification was $15 \mathrm{IU} / \mathrm{ml}$. HCV genotypes were identified using the HCV genotyping oligochip (Tianjin Third Central Hospital, Tianjin, China) as previously described (27).
METAVIR fibrosis stages. Needle biopsies of the liver were performed by a trained hepatologist, with a $16-\mathrm{G}$ cutting needle (Bard Peripheral Vascular, Inc., Tempe, AZ, USA). The samples were fixed in $10 \%$ formalin for $48 \mathrm{~h}$ at room temperature and embedded in paraffin. Liver tissue sections (4- $\mu \mathrm{m}$ thick) were stained with hematoxylin and eosin (H\&E) and Masson's trichrome at room temperature. For $\mathrm{H} \& \mathrm{E}$ staining the sections were deparaffinized and rehydrated with xylene and a decreasing graded ethanol series, stained in hematoxylin solution for $5 \mathrm{~min}$, differentiated in $0.5 \%$ acid alcohol for $30 \mathrm{sec}$ and stained blue in ammonia water. Counterstaining was performed in eosin solution for $1 \mathrm{~min}$. Masson's trichrome staining was performed in ponceau-picric acid saturated solution for $10 \mathrm{~min}$, rinsed in $1 \%$ acetic acid water, differentiated in $1 \%$ phosphomolybdic acid for $1 \mathrm{~min}$, rinsed in distilled water, stained in toluidine blue for $3 \mathrm{~min}$, rinsed in $1 \%$ acetic acid-water, differentiated in $95 \%$ alcohol, hydrated in absolute alcohol, cleared with xylene and mounted with neutral balsam. The liver sections were observed at x100 to x400 magnification using a Leica DM 2000 microscope (Leica Microsystems, Inc., Buffalo Grove, IL, USA).

All liver biopsies were evaluated by expert pathologists, who were blinded to the clinical history of the patients. Fibrosis was classified into five stages according to the METAVIR scoring system (28) as follows: 0, no fibrosis; 1, portal fibrosis without septa; 2 , portal fibrosis with rare septa; 3 , many septa without cirrhosis; and 4, cirrhosis. Liver fibrosis was evaluated according to the fibrosis METAVIR staging, with significant fibrosis defined as METAVIR stages $\geq 2$ (29).

Biochemical assays. Serum alanine aminotransferase (ALT) and AST were detected by optimized International Federation of Clinical Chemistry and Laboratory Medicine reference method (30), and total bilirubin (TBIL) was detected using the vanadate-oxidation method (31) with an Olympus AUS5400 automatic chemical analyzer (Olympus Corporation, Tokyo, Japan) according to the manufacturer's protocol. Blood platelet counts (PLT) were analyzed by an automated hematology analyzer using the Hydro Dynamic Focusing method (32) (XS-1000i; Sysmex Corporation, Kobe, Japan).

APRI and FIB-4 biomarker panels. APRI was calculated using the following formula: APRI=[AST (U/l)/upper limit of normal (U/1)] x100/PLT (10\%/1). The FIB-4 index was calculated using the following formula: FIB-4=Age (years)xAST (U/1)/PLT $\left(10^{9} / 1\right) x[\operatorname{ALT}(\mathrm{U} / 1)]^{1 / 2}(33)$.

LSM tests. Patients with CHC infection at baseline underwent LSM using FibroTouch (HISKY Medical Technologies Co., Ltd., Beijing, China) on the right lobe of the liver as previously described (34); the procedure was conducted by a technician who had performed >10 LSMs. A liver biopsy analysis was subsequently performed as described above. The results were expressed in kilopascals $(\mathrm{kPa})$ and the median value of 10 acquisitions was used for analysis, including only cases with a success rate $>60 \%$ and an interquartile range/median ratio of $<0.3$. The same professionally trained physician performed all of these procedures. 
Sample preparation. According to METAVIR fibrosis stages, 112 serum samples were collected from patients with $\mathrm{CHC}$ (genotype $1 \mathrm{~b}$ or $2 \mathrm{a}$ ) and fibrosis. Peripheral blood samples $(5 \mathrm{ml})$ were collected at the baseline and serum was separated by centrifugation at $1,200 \mathrm{x}$ g for $10 \mathrm{~min}$ at $4^{\circ} \mathrm{C}$. The supernatant was transferred into a new microcentrifuge tube and further processed by an additional centrifugation step at $12,000 \mathrm{x}$ g for $15 \mathrm{~min}$ at $4^{\circ} \mathrm{C}$ and stored at $-80^{\circ} \mathrm{C}$ prior to further analysis, as previously described (35).

RNA isolation and spike-in control. Total RNA was isolated from $250 \mu 1$ serum using TRIpure Reagent LS (Invitrogen; Thermo Fisher Scientific, Inc., Waltham, MA, USA) according to the manufacturer's protocol. For each RNA sample, the relative miR-1273g-3p expression levels were normalized to that of C. elegans miR-39-3p (cel-miR-39-3p; miRB0000010, Guangzhou RiboBio Co., Ltd., Guangzhou, China) because of its wide and stable expression in circulation $(36,37)$. Prior to RNA isolation, 40 fmol cel-miR-39 was added to each sample as a spike-in control. Total RNA was resuspended in nuclease-free, PCR-grade water and the RNA concentration was determined using the NanoDrop 2000C spectrophotometer (NanoDrop; Thermo Fisher Scientific Inc., Wilmington, DE, USA).

miR-1273g-3p quantification by RT-qPCR. Reverse transcription reactions were performed using the miScript-Reverse Transcription kit (Takara Biotechnology Co., Ltd., Dalian, China), cDNA was synthesized using reverse transcriptase with miR-1273g-3p specific stem-loop primers (forward primer, ssD1381210710; reverse primer, ssD089261711; RT-primer, ssD1381210709; Guangzhou RiboBio Co., Ltd.) and cel-miR-39-3p specific stem-loop primer (forward primer, ssD1083145002; reverse primer, ssD089261711; RT primer, ssD1083145001, Guangzhou RiboBio Co., Ltd.). The RT reaction was performed under the conditions of $37^{\circ} \mathrm{C}$ for $15 \mathrm{~min}$ and $85^{\circ} \mathrm{C}$ for $5 \mathrm{sec}$. Differential expression analysis was performed using RT-qPCR on an ABI 7500 Real-Time PCR system (Applied Biosystems; Thermo Fisher Scientific, Inc.) using SYBR Green Master Mix (Beijing CoWin Biotech Co., Ltd., Beijing, China). The amplification conditions were as follows: Initial denaturation at $95^{\circ} \mathrm{C}$ for $30 \mathrm{sec}$, then 40 cycles of $95^{\circ} \mathrm{C}$ for $5 \mathrm{sec}$ and $60^{\circ} \mathrm{C}$ for $30 \mathrm{sec}$. The relative abundance of miRNA was normalized to that of cel-miR-39 and the relative amount of each miRNA was measured using the $2^{-\Delta \Delta \mathrm{Cq}}$ method (38). All RT-qPCR reactions were conducted in triplicate. All data were obtained using Sequence Detector Software v2.0.4 (Applied Biosystems; Thermo Fisher Scientific Inc.).

Statistical analysis. Quantitative variables were expressed as the median (range) or mean \pm standard deviation and qualitative variables were expressed as percentages. Correlations between variables were calculated using Spearman rank order correlations and the diagnostic performance of non-invasive markers were evaluated by receiver operating characteristic (ROC) curves. Data were analyzed using SPSS software (version 16.0; SPSS, Inc., Chicago, IL, USA). All P-values were two-tailed and $\mathrm{P}<0.05$ was considered to indicate a statistically significant difference.

\section{Results}

Patient characteristics. The clinical characteristics of the cohort of 112 patients with CHC are summarized in Table I. There were 39 patients with mild liver fibrosis $(\mathrm{F}<2)$, including 17 males and 22 females, with a mean age of $43 \pm 12.42$ years. There were 47 subjects with moderate to severe liver fibrosis $(2 \leq \mathrm{F}<4)$, including 22 males and 25 females, with a mean age of $52.29 \pm 9.54$ years. There were 26 patients with cirrhosis $(\mathrm{F}=4)$, including 17 male and 9 female, with a mean age of $55.19 \pm 10.18$ years. There were no significant differences in patient sex, body mass index (BMI), HCV RNA load, HCV genotype, or ALT and AST levels at baseline between different grades of liver fibrosis. At higher fibrosis stages, the age $(\mathrm{P}<0.001)$ and TBIL $(\mathrm{P}=0.035)$ were significantly increased, whereas the PLT was decreased $(\mathrm{P}<0.001$; Table I). The different stages of fibrosis were assessed by histology and were distributed as follows among the samples: $\mathrm{F}<2,34.82 \%$; $2 \leq \mathrm{F}<4,41.96 \%$; and $\mathrm{F}=4,23.21 \%$.

Correlations between non-invasive models and histological findings. According to METAVIR fibrosis staging, patients were categorized as F1 to F4 as presented in Fig. 1. The Spearman's correlation coefficient results indicated that fibrosis stage was significantly correlated with miR-1273g-3p levels ( $\mathrm{r}=0.657, \mathrm{P}<0.001$; Fig. 2A). This correlation was lower compared with that between fibrosis stage and LSM $(r=0.815$, $\mathrm{P}<0.001$; Fig. 2B) and higher compared with that between fibrosis stage and APRI ( $\mathrm{r}=0.417, \mathrm{P}<0.001$; Fig. $2 \mathrm{C}$ ) or FIB-4 $(\mathrm{r}=0.522, \mathrm{P}<0.001$; Fig. 2D).

Performance of miR-1273g-3p, LSM, APRI and FIB-4 in fibrosis stage assessment. Patients were divided into three groups according to their METAVIR stage: $\mathrm{F}<2,2 \leq \mathrm{F}<4$ and $F=4$. The area under the ROC curve of miR-1273g-3p was 0.841 (95\% CI, 0.761-0.921) for the significant fibrosis and early fibrosis groups $(2 \leq \mathrm{F}<4$ and $\mathrm{F}<2$, respectively), which was lower compared with that of LSM $(0.890 ; 95 \%$ CI, 0.825-0.955), and higher compared with that of FIB-4 (0.791; 95\% CI, 0.701-0.881) and APRI (0.719; 95\% CI, 0.612-0.826; Fig. 3A; Table II). The sensitivity and specificity values of the four analyses are presented in Table II. Using an optimal cut-off of 2.67 , miR-1273g-3p had a sensitivity of $85 \%$ and specificity of $69 \%$ in predicting significant fibrosis. In addition, in ROC analysis of the performance of these markers in the diagnosis of cirrhosis $(\mathrm{F}=4$ vs. $\mathrm{F}<4)$, the AUC of miR-1273g-3p (0.933; 95\% CI, 0.874-0.993) was lower compared with that of LSM $(0.937 ; 95 \% \mathrm{CI}$, 0.887-0.987), and higher compared with values for FIB-4 (0.766; 95\% CI, 0.639-0.881) and APRI $(0.760 ; 95 \% \mathrm{CI}$, $0.649-0.871$ ). miR-1273g-3p had $80 \%$ sensitivity and $95 \%$ specificity for predicting cirrhosis with a cut-off value of 8.36 (Fig. 3B; Table III).

Predictive factors of miR-1273g-3p. The influence of certain factors on the expression of miR-1273g-3p was also assessed. It was observed that the serum levels of miR-1273g-3p were significantly positively correlated with age, BMI, and ALT, AST and TBIL levels ( $r=0.396,0.219,0.215,0.228$ and 0.225 , respectively; all $\mathrm{P}<0.05$; Fig. 4), whereas a significant negative 
Table I. Baseline characteristics of patients included in the present study $(n=112)$.

\begin{tabular}{|c|c|c|c|c|}
\hline Parameter & $\mathrm{F}<2$ & $2 \leq \mathrm{F}<4$ & $\mathrm{~F}=4$ & P-value \\
\hline Patients, n (\%) & $39(34.82)$ & 47 (41.96) & $26(23.21)$ & \\
\hline Sex, m/f & $17 / 22$ & $22 / 25$ & $17 / 9$ & 0.341 \\
\hline Age, years, mean \pm SD & $43 \pm 12.42$ & $52.29 \pm 9.54^{\mathrm{a}}$ & $55.19 \pm 10.18^{\mathrm{a}}$ & $<0.001$ \\
\hline $\mathrm{BMI}, \mathrm{kg} / \mathrm{m}^{2}$, mean $\pm \mathrm{SD}$ & $24.2 \pm 2.69$ & $25.39 \pm 3.12$ & $25.35 \pm 2.58$ & 0.21 \\
\hline ALT, U/l (range) & $31.5(9.0-199.0)$ & $33.0(5.0-363.0)$ & $38.4(13.0-345.0)$ & 0.199 \\
\hline AST, U/l (range) & $34.0(16.0-150.0)$ & $39.0(18.0-256.0)$ & $48.5(16.0-299.0)$ & 0.245 \\
\hline TBIL, $\mu \mathrm{mol} / 1$ (range) & $12.70(4.90-58.00)$ & $13.90(6.71-32.60)$ & $17.31(3.55-85.50)^{\mathrm{a}, \mathrm{b}}$ & 0.035 \\
\hline Platelets, $\times 10^{9} / 1$ (range) & $183.0(78.3-334.0)$ & $149.6(64.0-277.0)^{\mathrm{a}}$ & $125.0(31.0-304.0)^{\mathrm{a}, \mathrm{c}}$ & $<0.001$ \\
\hline HCV RNA, $\log _{10} \mathrm{IU} / \mathrm{ml}$ (range) & $6.02(4.48-7.99)$ & $6.31(3.60-7.84)$ & $6.15(3.63-7.18)$ & 0.532 \\
\hline HCV genotypes, $1 \mathrm{~b} / 2 \mathrm{a} / \mathrm{n} . \mathrm{d}$ & $22 / 11 / 6$ & $29 / 11 / 7$ & $8 / 8 / 10$ & 0.174 \\
\hline
\end{tabular}

${ }^{\mathrm{a}} \mathrm{P}<0.01$ vs. $\mathrm{F}<2$; ${ }^{\mathrm{P}}<0.01$ and ${ }^{\mathrm{c}} \mathrm{P}<0.05$ vs. $2 \leq \mathrm{F}<4$. $\mathrm{m}$, male; f, female; $\mathrm{SD}$, standard deviation; $\mathrm{BMI}$, body mass index; ALT, alanine aminotransferase; AST, aspartate aminotransferase; TBIL, total bilirubin; HCV, hepatitis C virus; n.d, not determined.

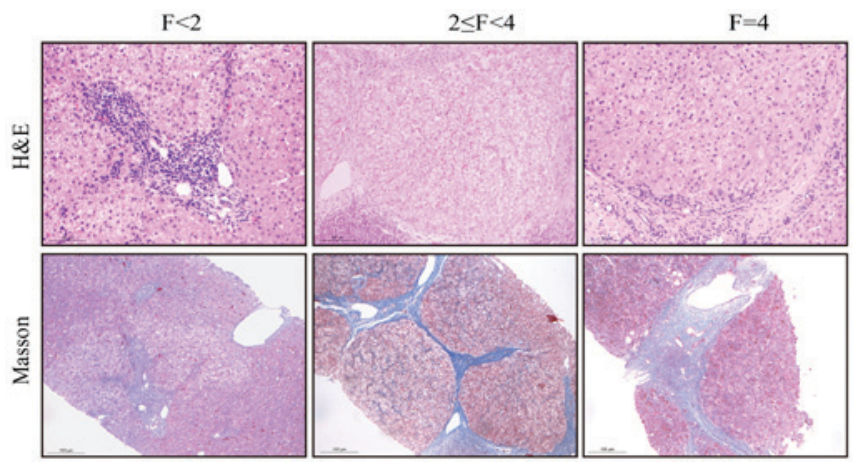

Figure 1. Histopathological changes in liver sections in patients with chronic hepatitis $\mathrm{C}$. The different stages of fibrosis were assessed by H\&E (magnification, x200) and Masson trichrome staining (magnification, x100). F values correspond to Meta-analysis of Histological Data in Viral Hepatitis scoring. $\mathrm{H} \& \mathrm{E}$, hematoxylin and eosin.

correlation with PLT count was observed ( $\mathrm{r}=-0.318 ; \mathrm{P}=0.001$; Fig. 4). No significant correlation was observed between miR-1273g-3p and baseline HCV RNA loads and genotype (Fig. 4).

\section{Discussion}

Liver biopsies are considered the gold standard for the diagnosis of liver fibrosis; however, this is an invasive procedure, which is subject to complications and high costs (39). Therefore, it is important to identify molecular markers that are able to predict disease progression. Increasing evidence suggests that miRNA profiling is a promising approach to facilitate the development of novel diagnostic biomarkers and the identification of therapeutic targets (40).

The results of a previous study by the present authors demonstrated that miR-1273g-3p was significantly upregulated in the fibrotic liver of patients with $\mathrm{CHC}$ and associated with higher fibrosis stages (41). In situ hybridization revealed that miR-1273g-3p was present in hepatocytes or hepatic stellate cells around the portal area (41). Additionally, it has been demonstrated that miR-1273g-3p modulates the
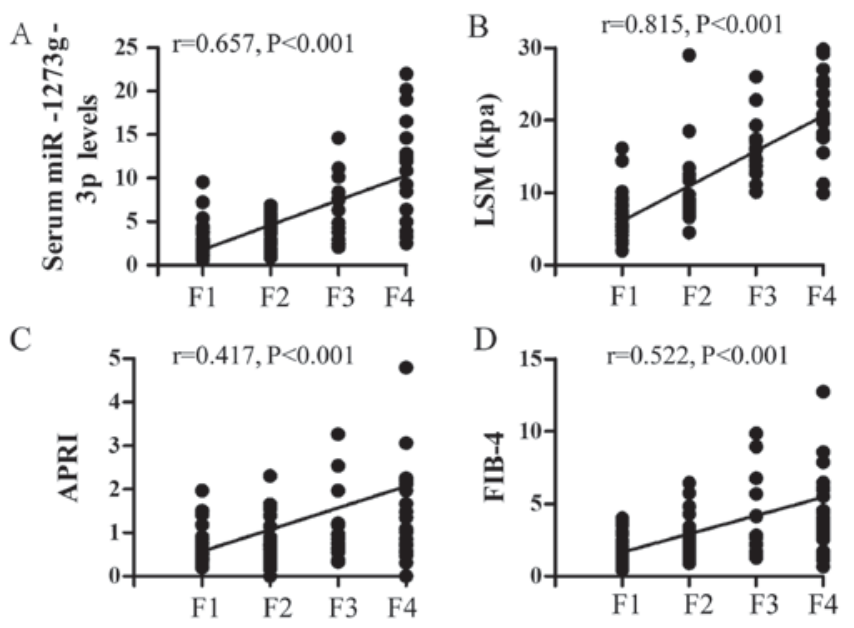

Figure 2. Correlations of miR-1273g-3p, LSM, APRI and FIB-4 with the fibrosis stage. (A) miR-1273g-3p, (B) LSM, (C) APRI and (D) FIB-4 were positively correlated with the stage of fibrosis. F values correspond to Meta-analysis of Histological Data in Viral Hepatitis scoring. miR, microRNA; LSM, liver stiffness measurements; APRI, aspartate aminotransferase-to-platelet ratio index; FIB-4, Fibrosis-4 score.

activation and apoptosis of hepatic stellate cells via phosphatase and tensin homologs in $\mathrm{HCV}$-associated liver fibrosis. The effects of various miRNAs on liver fibrosis have been illustrated in previous studies (11,41-43). The miR-199 and -200 families are upregulated with the progression of liver fibrosis (44). miR-29 family members, including miR-29b and miR-29c, have been reported to be associated with the occurrence of fibrosis via regulating the synthesis of extracellular matrix components, particularly collagen (45). Previous studies have demonstrated that hepatic levels of miR-122 decrease significantly as the severity of fibrosis increases $(14,42)$. miRNA levels in HCC samples with or without previous HCV infection have also been reported; thus, determination of HCV-specific effects in these studies is complicated by the overall dysregulation of miRNAs observed in tumors (46). Taken together, changes in miRNA expression appear to be sensitive indicators of hepatic injury 
Table II. Validation and comparison of non-invasive methods for the prediction of liver fibrosis in chronic hepatitis C (Meta-analysis of Histological Data in Viral Hepatitis stage F2-4 vs. F1).

\begin{tabular}{lccccr}
\hline Assay & AUC & $95 \%$ CI & Cut-off & Se & Sp \\
\hline miR-1273g-3p & 0.841 & $0.761-0.921$ & 2.67 & 0.85 & 0.69 \\
LSM & 0.890 & $0.825-0.955$ & 9.50 & 0.70 & 0.92 \\
APRI & 0.719 & $0.612-0.826$ & 0.55 & 0.78 & 0.69 \\
FIB-4 & 0.791 & $0.701-0.881$ & 2.49 & 0.66 & 0.83 \\
\hline
\end{tabular}

miR, microRNA; LSM, liver stiffness measurement; APRI, aspartate aminotransferase-to-platelet count ratio index; FIB-4, fibrosis-4; AUC, area under curve; CI, confidence interval; Se, sensitivity; Sp, specificity.
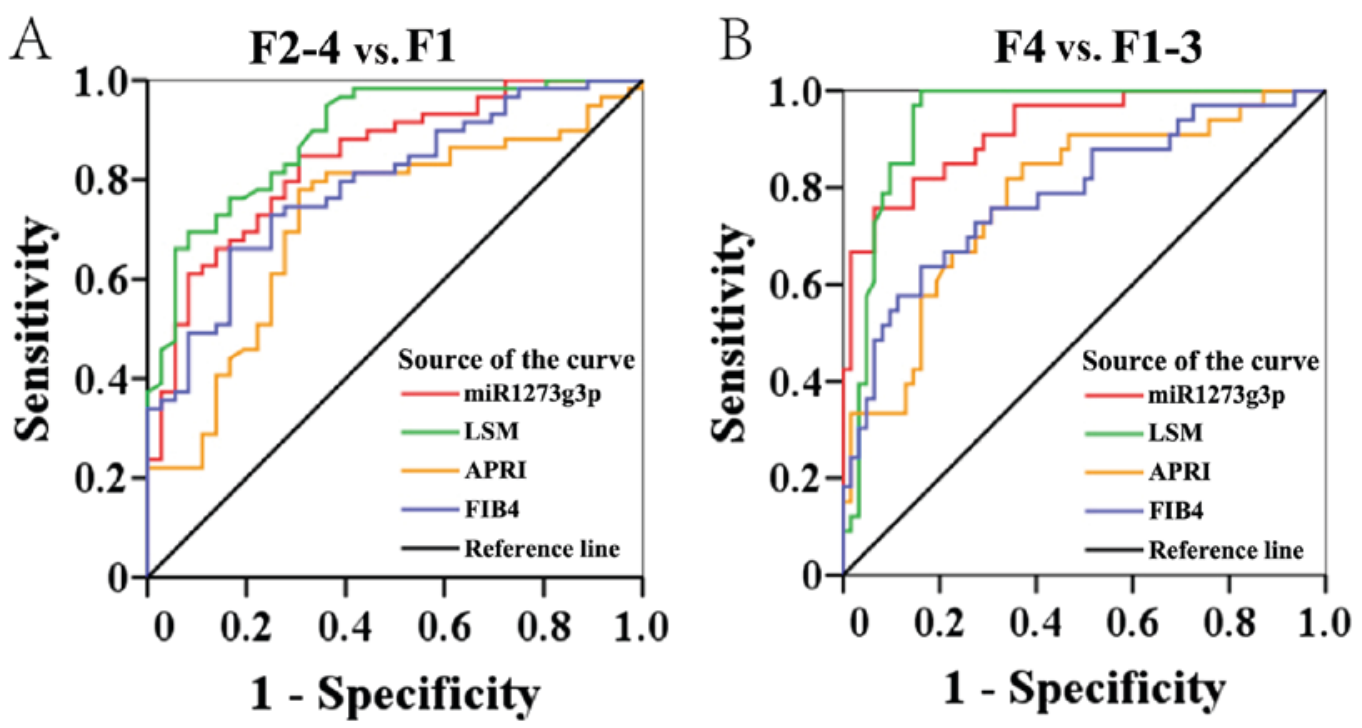

Figure 3. Receiver operating characteristic curve analysis of serum miR-1273g-3p, LSM, APRI and FIB-4 in distinguishing (A) $4>F \geq 2$ from F1, and (B) F=4 from F1-3. F values correspond to Meta-analysis of Histological Data in Viral Hepatitis scoring. miR, microRNA; LSM, liver stiffness measurements; APRI, aspartate aminotransferase-to-platelet ratio index; FIB-4, Fibrosis-4 score; $4>\mathrm{F} \geq 2$, significant liver fibrosis; F1, non-significant liver fibrosis; $\mathrm{F}=4$, cirrhosis.

and are potentially associated with the development of liver fibrosis (14).

In the present study, the pathological results of patients with $\mathrm{CHC}$ were used as reference standards, and additional analyses were performed to evaluate the association between miR-1273g-3p and LSM, APRI and FIB-4. Acoustic radiation force impulse, APRI and FIB-4 have been previously reported to be elevated in patients with chronic hepatitis $B$ infection and correlated with the stage of fibrosis (47). In the present study, levels of miR-1273g-3p were increased with the stage of liver fibrosis, and the correlation coefficient between fibrosis stage and miR-1273g-3p was higher compared with that for APRI and FIB-4, whereas it was lower compared with that for LSM. The results of the present study demonstrate that miR-1273g-3p, LSM, APRI and FIB-4 may be used to determine the stage of fibrosis according to the METAVIR score. The potential of miR-1273g-3p as an indicator of fibrosis progression in $\mathrm{CHC}$ was evaluated. ROC analysis demonstrated that the performance of miR-1273g-3p in differentiating between $F=4$ and $4>\mathrm{F} \geq 2$ stages was superior compared with that of FIB-4 and APRI; however, it was inferior compared with that of LSM for the diagnosis of significant fibrosis. The cut-off value of miR-1273g-3p was increased with the stage of fibrosis, suggesting that cut-off values may be used to predict the stage of fibrosis. Our previous study demonstrated that, compared with APRI and FIB-4, LSM is a more convenient and reliable diagnostic indicator of liver fibrosis in patients with chronic liver disease (20). However, LSM may be affected by liver inflammation, increased transaminase levels and TBIL.

Non-invasive assessment of liver fibrosis is an important goal for the treatment of patients with $\mathrm{CHC}$ (48). In the present study, miR-1273g-3p levels were low in the early stages and increased in the later stages of fibrosis $(F \geq 2)$, suggesting a changing pattern of circulating miR-1273g-3p levels as the disease progresses. Further analysis of the factors that affect the miR-1273g-3p expression levels revealed that age, BMI, and ALT, AST and TBIL levels were positively correlated with miR-1273g-3p expression, whereas a negative correlation was observed between miR-1273g-3p expression and PLT. These results indicate that the expression of miR-1273g-3p may be affected by liver inflammation, increased transaminase levels, TBIL and PLT. 
Table III. Validation and comparison of non-invasive models for the prediction of liver fibrosis in chronic hepatitis C (Meta-analysis of Histological Data in Viral Hepatitis F4 vs. F1-3).

\begin{tabular}{lccrrr}
\hline Assay & AUC & $95 \%$ CI & Cut-off & Se & Sp \\
\hline miR-1273g-3p & 0.933 & $0.874-0.993$ & 8.36 & 0.80 & 0.95 \\
LSM & 0.937 & $0.887-0.987$ & 15.09 & 0.90 & 0.89 \\
APRI & 0.760 & $0.649-0.871$ & 0.67 & 0.85 & 0.63 \\
FIB-4 & 0.766 & $0.639-0.881$ & 2.95 & 0.80 & 0.75 \\
\hline
\end{tabular}

miR, microRNA; LSM, liver stiffness measurement; APRI, aspartate aminotransferase-to-platelet count ratio index; FIB-4, fibrosis-4; AUC, area under curve; CI, confidence interval; Se, sensitivity; Sp, specificity.
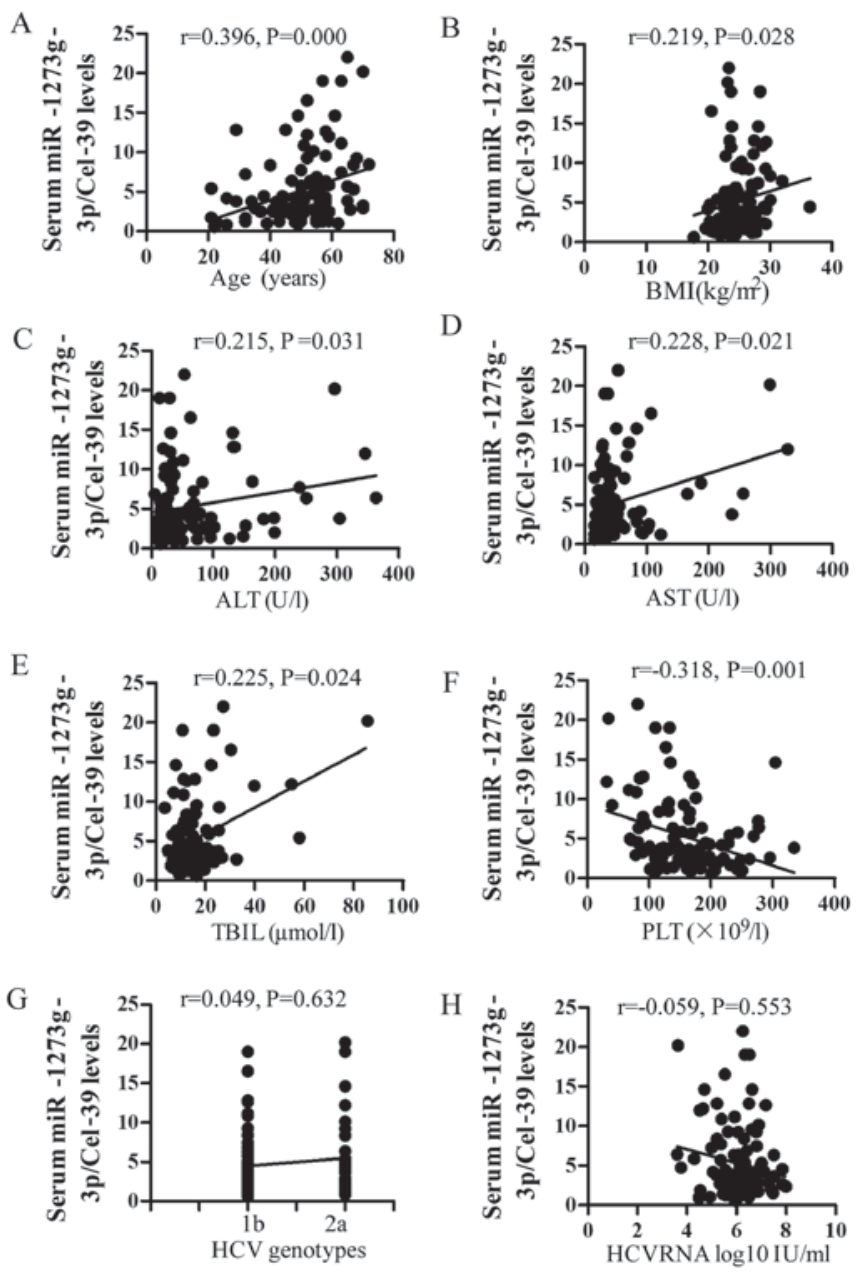

Figure 4. Correlation between miR-1273g-3p, baseline characteristics and biochemical assays. The Spearman analysis demonstrated a positive correlation between miR-1273g-3p and (A) age, (B) BMI, (C) ALT, (D) AST and (E) TBIL and a negative correlation between miR-1273g-3p and (F) PLT. No correlation was observed between miR-1273g-3p and (G) HCV RNA viral load or (H) HCV genotype. miR, microRNA; BMI, body mass index; ALT, alanine aminotransferase; AST, aspartate aminotransferase; TBIL, total bilirubin; $\mathrm{HCV}$, hepatitis $\mathrm{C}$ virus.

Several limitations of the present study should be noted. The study was conducted at a single center and the small proportion of patients with significant fibrosis may be a source of selection bias. To corroborate these results, further studies involving large patient populations are required to analyze the association between serum miR-1273g-3p levels and HCV-induced hepatic steatosis, inflammation and cholestasis, as well as other etiologies of liver disease.

\section{Acknowledgements}

Not applicable.

\section{Funding}

The present study was supported by the Chinese Foundation for Hepatitis Prevention and Control's Wang Bao-en Hepatic Fibrosis Foundation (grant no. 20140018), the Graduate Student Innovation Fund Project in Hebei Province (2015), the Key Science and Technology Project of Hebei Province (grant no. 14277746D), the Hebei Province Key Laboratory of Research and Development for Chinese Medicine (2014) and the Government-Funded Clinical Medical Talents Projects in Hebei Province (2016).

\section{Availability of data and materials}

All data generated or analyzed during this study are included in this published article.

\section{Authors' contributions}

YN designed the research; XN, NF, JD and BW performed the experiments; $\mathrm{YW}, \mathrm{YZ}$ and $\mathrm{SZ}$ analyzed data; $\mathrm{XN}, \mathrm{RW}$ and $\mathrm{YN}$ wrote the paper.

\section{Ethics approval and consent to participate}

Written informed consent was obtained from all patients and the study was approved by the Ethics Committee of the Third Hospital of Hebei Medical University, according to the Declaration of Helsinki and Good Clinical Practice guidelines.

\section{Consent for publication}

Not applicable.

\section{Competing interests}

The authors declare that they have no competing interests. 


\section{References}

1. Wilkins T, Akhtar M, Gititu E, Jalluri C and Ramirez J: Diagnosis and management of hepatitis C. Am Fam Phys 91: 835-842, 2015

2. WHO guidelines approved by the guidelines review committee: In: Guidelines for the screening, care and treatment of persons with hepatitis C infection, Geneva 2014.

3. Kayadibi H, Yasar B, Ozkara S, Serdar MA, Kurdas OO and Gonen C: The diagnostic accuracy of the Forns index, platelet count and AST to platelet ratio index derived fibrosis index for the prediction of Hepatitis C virus-related significant liver fibrosis and cirrhosis. Scand J Clin Lab Invest 74: 240-247, 2014.

4. Ghany MG, Strader DB, Thomas DL and Seeff LB; American Association for the Study of Liver Diseases: Diagnosis, management, and treatment of hepatitis C: An update. Hepatology 49: $1335-1374,2009$

5. Bravo AA, Sheth SG and Chopra S: Liver biopsy. N Engl J Med 344: 495-500, 2001

6. Noetel A, Kwiecinski M, Elfimova N, Huang J and Odenthal M: microRNA are central players in anti- and profibrotic gene regulation during liver fibrosis. Front Physiol 3: 49, 2012.

7. Kosaka N, Iguchi $\mathrm{H}$ and Ochiya T: Circulating microRNA in body fluid: A new potential biomarker for cancer diagnosis and prognosis. Cancer Sci 101: 2087-2092, 2010.

8. Lee HM, Nguyen DT and Lu LF: Progress and challenge of microRNA research in immunity. Front Genet 5: 178, 2014.

9. Othman $\mathrm{N}$ and Nagoor $\mathrm{NH}$ : The role of microRNAs in the regulation of apoptosis in lung cancer and its application in cancer treatment. Biomed Res Int 2014: 318030, 2014.

10. Shenoy A and Blelloch RH: Regulation of microRNA function in somatic stem cell proliferation and differentiation. Nat Rev Mol Cell Biol 15: 565-576, 2014.

11. Du J, Niu X, Wang Y, Kong L, Wang R, Zhang Y, Zhao S and Nan Y: MiR-146a-5p suppresses activation and proliferation of hepatic stellate cells in nonalcoholic fibrosing steatohepatitis through directly targeting Wnt1 and Wnt5a. Sci Rep 5: 16163 , 2015.

12. Abdollahi M, Pouri A, Ghojazadeh M, Estakhri R and Somi M Non-invasive serum fibrosis markers: A study in chronic hepatitis. Bioimpacts 5: 17-23, 2015.

13. Bala S, Petrasek J, Mundkur S, Catalano D, Levin I, Ward J, Alao H, Kodys K and Szabo G: Circulating microRNAs in exosomes indicate hepatocyte injury and inflammation in alcoholic, drug-induced, and inflammatory liver diseases. Hepatology 56: 1946-1957, 2012.

14. Halász T, Horváth G, Pár G, Werling K, Kiss A, Schaff Z and Lendvai G: miR-122 negatively correlates with liver fibrosis as detected by histology and FibroScan. World J Gastroenterol 21: 7814-7823, 2015.

15. Mahgoub A and Steer CJ: MicroRNAs in the evaluation and potential treatment of liver diseases. J Clin Med 5: E52, 2016.

16. Cortez MA and Calin GA: MicroRNA identification in plasma and serum: A new tool to diagnose and monitor diseases. Expert Opin Biol Ther 9: 703-711, 2009.

17. Cortez MA, Bueso-Ramos C, Ferdin J, Lopez-Berestein G, Sood AK and Calin GA: MicroRNAs in body fluids-the mix of hormones and biomarkers. Nat Rev Clin Oncol 8: 467-477, 2011.

18. Etheridge A, Lee I, Hood L, Galas D and Wang K: Extracellular microRNA: A new source of biomarkers. Mutat Res 717: 85-90, 2011.

19. Castera L, Winnock M, Pambrun E, Paradis V, Perez P, Loko MA, Asselineau J, Dabis F, Degos F and Salmon D: Comparison of transient elastography (FibroScan), FibroTest, APRI and two algorithms combining these non-invasive tests for liver fibrosis staging in $\mathrm{HIV} / \mathrm{HCV}$ coinfected patients: ANRS CO13 HEPAVIH and FIBROSTIC collaboration. HIV Med 15: 30-39, 2014.

20. Wang R, Ren W, Zhao S, Niu X, Tan P, Du H and Nan Y: Clinical study on FibroTouch and multi-parameter model for diagnosis of hepatic fibrosis in patients with chronic liver disease. Zhonghua Gan Zang Bing Za Zhi 23: 265-269, 2015 (In Chinese).

21. Crespo G, Fernández-Varo G, Mariño Z, Casals G, Miquel R, Martínez SM, Gilabert R, Forns X, Jiménez W and Navasa M: ARFI, FibroScan, ELF, and their combinations in the assessment of liver fibrosis: A prospective study. J Hepatol 57: 281-287, 2012.
22. Friedrich-Rust M, Ong MF, Martens S, Sarrazin C, Bojunga J, Zeuzem S and Herrmann E: Performance of transient elastography for the staging of liver fibrosis: A meta-analysis. Gastroenterology 134: 960-974, 2008.

23. Fraquelli M, Rigamonti C, Casazza G, Donato MF, Ronchi G, Conte D, Rumi M,Lampertico P and Colombo M: Etiology-related determinants of liver stiffness values in chronic viral hepatitis B or C. J Hepatol 54: 621-628, 2011.

24. Baranova A, Lal P, Birerdinc A and Younossi ZM: Non-invasive markers for hepatic fibrosis. BMC Gastroenterol 11: 91, 2011.

25. European Association for the Study of the Liver: EASL clinical practice guidelines: Management of hepatitis $\mathrm{C}$ virus infection. J Hepatol 55: 245-264, 2011.

26. Chinese Society of Hepatology, Chinese Society of Infectious Diseases, Parasitology of Chinese Medical Association. The guideline for prevention and treatment of hepatitis C. Zhonghua Gan Zang Bing Za Zhi 12: 194-198, 2004 (In Chinese).

27. Sun ZH, Yang HL, Wei M, Wang SY, Wang CR, Shi YL and Ma WL: Preparation and application of oligo microarrays for hepatitis virus detection and genotyping. Zhonghua Gan Zang Bing Za Zhi 15: 816-820, 2007 (In Chinese).

28. Intraobserver and interobserver variations in liver biopsy interpretation in patients with chronic hepatitis $\mathrm{C}$. The French METAVIR Cooperative Study Group. Hepatology 20: 15-20, 1994.

29. Amorim TG, Staub GJ, Lazzarotto C, Silva AP, Manes J, Ferronato Mda G, Shiozawa MB, Narciso-Schiavon JL, Dantas-Correa EB and Schiavon Lde L: Validation and comparison of simple noninvasive models for the prediction of liver fibrosis in chronic hepatitis C. Ann Hepatol 11: 855-861, 2012.

30. Schumann G, Bonora R, Ceriotti F, Férard G, Ferrero CA, Franck PF, Gella FJ, Hoelzel W, Jørgensen PJ, Kanno T, et al: IFCC primary reference procedures for the measurement of catalytic activity concentrations of enzymes at 37 degrees $\mathrm{C}$. International federation of clinical chemistry and laboratory medicine. Part 4. Reference procedure for the measurement of catalytic concentration of alanine aminotransferase. Clin Chem Lab Med 40: 718-724, 2002.

31. Ye YW, Wang YS and Shen ZY: National guide to clinical laboratory procedures. 3rd edition. Southeast University Press, Nanjing, 2006 (In Chinese).

32. Hill VL, Simpson VZ, Higgins JM, Hu Z, Stevens RA, Metcalf JA and Baseler M: Evaluation of the performance of the Sysmex XT-2000i hematology analyzer with whole bloods stored at room temperature. Lab Med 40: 709-718, 2009.

33. Teshale E, Lu M, Rupp LB, Holmberg SD, Moorman AC, Spradling P, Vijayadeva V, Boscarino JA, Schmidt MA and Gordon SC; CHeCS Investigators: APRI and FIB-4 are good predictors of the stage of liver fibrosis in chronic hepatitis B: The Chronic Hepatitis Cohort Study (CHeCS). J Viral Hepat 21: 917-920, 2014.

34. Castéra L, Vergniol J, Foucher J, Le Bail B, Chanteloup E, Haaser M, Darriet M, Couzigou P and De Lédinghen V: Prospective comparison of transient elastography, Fibrotest, APRI, and liver biopsy for the assessment of fibrosis in chronic hepatitis C. Gastroenterology 128: 343-350, 2005.

35. Mummadi RR, Petersen JR, Xiao SY and Snyder N: Role of simple biomarkers in predicting fibrosis progression in $\mathrm{HCV}$ infection. World J Gastroenterol 16: 5710-5715, 2010.

36. Kroh EM, Parkin RK, Mitchell PS and Tewari M: Analysis of circulating microRNA biomarkers in plasma and serum using quantitative reverse transcription-PCR (qRT-PCR). Methods 50: 298-301, 2010.

37. Arroyo JD, Chevillet JR, Kroh EM, Ruf IK, Pritchard CC Gibson DF, Mitchell PS, Bennett CF, Pogosova-Agadjanyan EL, Stirewalt DL, et al: Argonaute2 complexes carry a population of circulating microRNAs independent of vesicles in human plasma. Proc Natl Acad Sci USA 108: 5003-5008, 2011.

38. Livak KJ and Schmittgen TD: Analysis of relative gene expression data using real-time quantitative PCR and the 2(-Delta Delta C(T)) method. Methods 25: 402-408, 2001

39. Bedossa P, Dargère D and Paradis V: Sampling variability of liver fibrosis in chronic hepatitis C. Hepatology 38: 1449-1457, 2003.

40. Yokoi T and Nakajima M: Toxicological implications of modulation of gene expression by microRNAs. Toxicol Sci 123: 1-14, 2011

41. Niu X, Fu N, Du J, Wang R, Wang Y, Zhao S, Du H, Wang B, Zhang Y, Sun D and Nan Y: miR-1273g-3p modulates activation and apoptosis of hepatic stellate cells by directly targeting PTEN in HCV-related liver fibrosis. FEBS Lett 590: 2709-2724, 2016 
42. Trebicka J, Anadol E, Elfimova N, Strack I, Roggendorf M, Viazov S, Wedemeyer I, Drebber U, Rockstroh J, Sauerbruch T, et al: Hepatic and serum levels of miR-122 after chronic HCV-induced fibrosis. J Hepatol 58: 234-239, 2013.

43. Wang J, Chu ES, Chen HY, Man K, Go MY, Huang XR, Lan HY, Sung JJ and Yu J: microRNA-29b prevents liver fibrosis by attenuating hepatic stellate cell activation and inducing apoptosis through targeting PI3K/AKT pathway. Oncotarget 6: 7325-7338, 2015.

44. Murakami Y, Toyoda H, Tanaka M, Kuroda M, Harada Y Matsuda F, Tajima A, Kosaka N, Ochiya T and Shimotohno K The progression of liver fibrosis is related with overexpression of the miR-199 and 200 families. PloS One 6: e16081, 2011.

45. Roderburg C, Urban GW, Bettermann K, Vucur M, Zimmermann H, Schmidt S, Janssen J, Koppe C, Knolle P, Castoldi M, et al: Micro-RNA profiling reveals a role for miR-29 in human and murine liver fibrosis. Hepatology 53: 209-218, 2011.
46. Zekri AN, Youssef AS, El-Desouky ED, Ahmed OS, Lotfy MM, Nassar AA and Bahnassey AA: Serum microRNA panels as potential biomarkers for early detection of hepatocellular carcinoma on top of HCV infection. Tumour Biol 37: 12273-12286, 2016.

47. Liu J, Liu Y, Dong C, Yao S, Li S, Yuan J, Chen C, Zhao M, Lin Y and Peng Z: ARFI, Forns index, FIB-4 and APRI diagnosis liver fibrosis in patients with chronic liver diseases. Zhongguo Gan Zang Bing Za Zhi 6: 18-21, 2014 (In Chinese).

48. Vergniol J, Boursier J, Coutzac C, Bertrais S, Foucher J, Angel C, Chermak F, Hubert IF, Merrouche W, Oberti F, et al: Evolution of noninvasive tests of liver fibrosis is associated with prognosis in patients with chronic hepatitis C. Hepatology 60: 65-76, 2014. 